\title{
Self-Assembly of Porous Structures From a Binary Mixture of Lobed Patchy Particles
}

\author{
Sanjib Paul ${ }^{1}$ and Harish Vashisth ${ }^{2 *}$ \\ ${ }^{1}$ Department of Chemistry, New York University, New York, NY, United States, ${ }^{2}$ Department of Chemical Engineering, University \\ of New Hampshire, Durham, NH, United States
}

We report simulation studies on the self-assembly of a binary mixture of snowman and dumbbell shaped lobed particles. Depending on the lobe size and temperature, different types of self-assembled structures (random aggregates, spherical aggregates, liquid droplets, amorphous wire-like structures, amorphous ring structures, crystalline structures) are observed. At lower temperatures, heterogeneous structures are formed for lobed particles of both shapes. At higher temperatures, homogeneous self-assembled structures are formed mainly by the dumbbell shaped particles, while the snowman shaped particles remain in a dissociated state. We also investigated the porosities of self-

OPEN ACCESS

Edited by:

Ramon Castañeda-Priego, University of Guanajuato, Mexico

Reviewed by: Gerardo Odriozola, Autonomous Metropolitan University, Mexico

Jose Antonio Moreno-Razo, Autonomous Metropolitan University, Mexico

*Correspondence: Harish Vashisth harish.vashisth@unh.edu

Specialty section: This article was submitted to Soft Matter Physics,

a section of the journal

Frontiers in Physics

Received: 31 August 2021 Accepted: 30 September 2021

Published: 19 October 2021

Citation:

Paul S and Vashisth H (2021) SelfAssembly of Porous Structures From a

Binary Mixture of Lobed Patchy Particles.

Front. Phys. 9:767623.

doi: 10.3389/fphy.2021.767623 assembled structures. The pore diameters in self-assemblies increased with an increase in temperature for a given lobe size. The particles having smaller lobes produced structures with larger pores than the particles having larger lobes. We further investigated the effect of $\sigma$, a parameter in the surface-shifted Lennard-Jones potential, on the self-assembled morphologies and their porosities. The self-assembled structures formed at a higher $\sigma$ value are found to produce larger pores than those at a lower $\sigma$.

Keywords: lobed particles, self-assembly, porous structures, particle mixtures, Langevin dynamics

\section{INTRODUCTION}

The spherical colloidal particles usually self-assemble into close-packed structures driven by their isotropic interactions [1]. On the contrary, colloidal particles with patches, the patchy particles, can self-assemble into diverse structures due to anisotropic interactions [2]. Therefore, the synthesis and the self-assembly behavior of patchy particles are being increasingly investigated in the materials science research [3-19], because these particles provide a way to fabricate unique morphologies on a nanometer to micrometer scale for various technological and biomedical applications [20-25].

Although several methods have been developed to produce spherical or non-spherical patchy particles, producing a homogeneous mixture of a single type of particles remains challenging [26-29]. As a result, studies are needed to probe the self-assembly behavior of mixtures of different types of particles. In our earlier studies [30-33], we reported on self-assembled morphologies obtained from different types of lobed patchy particles where the patches around a central seed sphere appear as protrusions or lobes. Specifically, we showed that unique porous self-assembled morphologies can be produced depending on the number and the location of lobes. We reported that the dumbbell shaped particles, having two lobes at two opposite poles, can produce larger pores than the particles with higher number of lobes [31]. In addition, our earlier study on the effect of the lobe size and temperature on the self-assembly showed that the single-lobed snowman shaped particles self-assemble into only sheets or micelle like structures, whereas the dumbbell shaped particles can 
self-assemble into different non-crystalline and crystalline structures at different lobe sizes and temperatures. Moreover, the dumbbell shaped particles self-assemble at a wider range of temperature and lobe size, but the snowman shaped particles selfassemble only at higher lobe sizes and lower temperatures. In the present work, we aim to study the self-assembled morphologies and porosities obtained from a binary mixture of these two types of particles. A binary mixture of these lobed particles, that have only fewer number of lobes, is expected to enrich the spectrum of the morphologies. For example, an earlier theoretical study showed that a large part of the phase space of the selfassembled structures formed by a binary mixture of patchy particles having very fewer number of patches are occupied by empty liquids [34].

Among experimental methods to produce patchy particles, Park et al. [35] developed a method of producing monodispersed snowman shaped particles (in their work, they refer the particles having two spheres as dumbbell-shaped particles) on the micrometer scale using seeded-emulsion polymerization technique. The dumbbell shaped particles, that they produced at different diameter ratios of two spheres, self-assembled into thin two-dimensional layers. Using the same seeded-emulsion polymerization method, Peng et al. [36] synthesized monodisperse snowman and dumbbell shaped particles. Wang et al. [5] developed a method of making non-spherical particles having not only one or two lobes, but more than two lobes. At first, by making the clusters of different shapes using polystyrene beads, and then using a two-stage swelling and polymerization technique, they produced particles of seven different shapes. They showed that a binary mixture of snowman and dumbbell shaped particles, where the lobes of each kind of particles are functionalized with complementary DNA strands, selfassemble into small linear chains at smaller lobe sizes. When the lobes are smaller and the amount of single-lobed snowman shaped particles is less, they form cis-trans-like isomers. When the amount of snowman shaped particles is increased, they form ethylene like molecules where a dumbbell shaped particle using its two lobes remains attached with four snowman shaped particles.

In our work, we studied the self-assembled morphologies produced by a mixture of the snowman and dumbbell shaped particles at different lobe sizes. An earlier study [5] showed that the mixtures of these two types of particles self-assemble into different small clusters at different conditions. Andre et al. [29] showed that the dumbbell and snowman-shaped soft patchy particles at nanometer scale range can co-assemble into colloidal polymers of several micrometers. However, the larger clusters, that can be obtained from the co-assembly of the snowman and dumbbell shaped hard particles, have not been reported yet but such structures would find practical technological or biological applications [37, 38]. To probe these questions, we studied a binary mixture of the snowman and dumbbell shaped lobed particles where we have varied several parameters including the lobe size, temperature, and the $\sigma$ in the shifted Lennard-Jones potential, and observed their effect on self-assembled morphologies and their functional properties such as porosities.

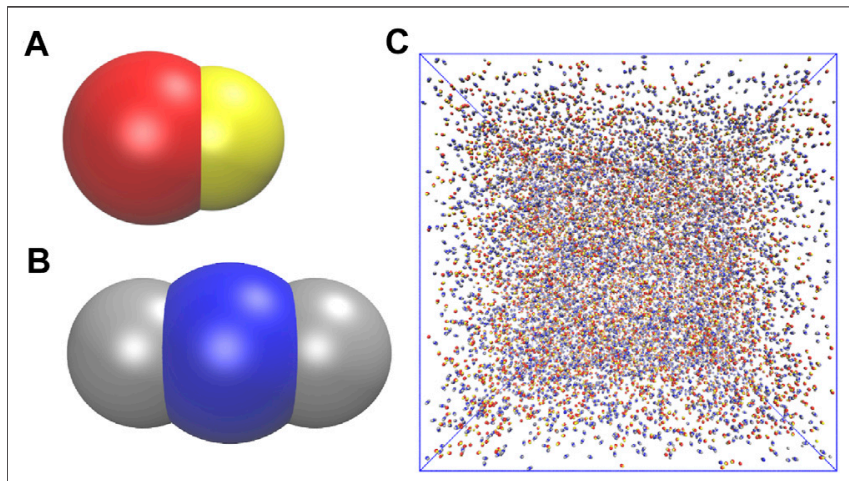

FIGURE 1 | (A) A snowman shaped and (B) a dumbbell shaped particle. The seed and the lobe of the snowman shaped particle are represented in red and yellow color respectively. The seed and the lobes of the dumbbell shaped particle are represented in blue and gray color respectively. (C) A representation of the simulation domain showing a disassembled mixture of snowman and dumbbell shaped particles.

\section{MODELS AND METHODS}

We studied the self-assembly of a mixture of snowman and dumbbell shaped particles. To model these particles, we followed the approach reported in our previous work [31]. For each dumbbell shaped particle, we placed two spherical beads (Supplementary Figure S1) each with a diameter $\left(\sigma_{\mathrm{B}}\right)$ of 1.0 (in all of our simulations, $\sigma_{\mathrm{B}}$ is the unit of distance) at a distance of $1.0 \sigma_{\mathrm{B}}$, that is equal to the sum of their radius $\left(1.0 \sigma_{\mathrm{B}}\right)$, meaning that two spherical beads touch each other. We then placed another spherical bead (the diameter of which is denoted by $\sigma_{\mathrm{S}}$ ) at the center of mass of these two spherical beads. Therefore, the distance between the central bead and any of the terminal beads is $0.5 \sigma_{\mathrm{B}}$. Following our previous work [31], we refer to the central bead as a seed. After inserting the seed, the exposed parts of the terminal beads, act as lobes. For the snowman-shaped particles, we first placed two spherical beads at a $0.5 \sigma_{\mathrm{B}}$ distance apart. One of these two spherical beads acts as the seed and the exposed part of another bead acts as the lobe. For both types of particles, we gradually changed the seed diameter $\left(\sigma_{\mathrm{S}}\right)$ from $1.0 \sigma_{\mathrm{B}}$ to $2.0 \sigma_{\mathrm{B}}$ to vary the lobe size, and fixed the diameter of the beads which act as lobes. The higher the diameter of the seed, lower is the lobe size (Supplementary Figures S1B-G). We quantify the lobe size by a parameter termed $q_{\mathrm{L}}$ which is the ratio of $\sigma_{\mathrm{B}}$ to $\sigma_{\mathrm{S}}$ $\left(q_{\mathrm{L}}=\sigma_{\mathrm{B}} / \sigma_{\mathrm{S}}\right)$. As we increase the seed diameter $\left(\sigma_{\mathrm{S}}\right), q_{\mathrm{L}}$ decreases from 1.0 to $0.50\left(q_{\mathrm{L}}=1.0,0.83,0.71,0.63,0.56,0.50\right)$. The opening angle $(\delta)$ of the lobes (Supplementary Figure $\mathbf{S 1 H}$ ), which is defined by the equation (39) $\delta=\left(\sigma_{\mathrm{B}}^{2}-\sigma_{\mathrm{S}}^{2}-4 d^{2}\right) / 4 d \sigma_{\mathrm{S}}$, where $d$ is equal to $1 / 2 \sigma_{\mathrm{B}}$, the distance between the central seed and a lobe, decreases from $120^{\circ}$ to $0^{\circ}\left(\delta=120^{\circ}, 106.27^{\circ}, 91.15^{\circ}, 73.75^{\circ}, 51.69^{\circ}\right.$, $0^{\circ}$ ) with the increase of the seed diameter or the decrease of the lobe size.

We performed coarse grained Langevin dynamics simulations in constant volume and temperature ensemble using the HOOMD-Blue software [40, 41]. We studied the self-assembly of the mixture at three different number ratios $\left(r_{\mathrm{N}}=N_{\mathrm{S}} / N_{\mathrm{D}}\right.$; where $N_{\mathrm{S}}$ and $N_{\mathrm{D}}$ is the number of the snowman and dumbbell 
shaped particles, respectively) of the lobed particles $\left(r_{\mathrm{N}}=0.5,1.0\right.$, and 2.0). When $r_{\mathrm{N}}$ is equal to 1.0, the simulation domain contains 8,000 lobed particles of each type, thereby totaling 16,000 particles. At $r_{\mathrm{N}}=0.5$ or 2.0 , the number of particles of one type is doubled keeping the number of particles of the other type fixed at 8,000. Therefore, the total number of the particles at $r_{\mathrm{N}}=$ 0.5 and 2.0 is 24,000 . In all of our simulations, the length of the simulation domain is $140 \sigma_{\mathrm{B}}$ in each direction.

The size of a seed and a lobe is same for each particle present in the simulation domain. In Figure 1, we show a snowman and a dumbbell shaped particle of $\sigma_{S}=1.2$ and an initial simulation domain of the binary mixture where the lobed particles are in a dissociated state. We studied the self-assembly at six different seed diameters, and for each seed diameter, we varied the temperature $\left(k_{B} T\right)$ from 0.1 to $0.5\left(k_{B} T=0.1,0.2,0.3,0.4,0.5\right)$. For each simulation, we first obtained a randomized initial configuration by performing Langevin dynamics simulation for $10^{5}$ steps at a higher temperature $\left(k_{B} T=1.5\right)$. We used the randomized initial configuration of each system to study the self-assembly at a desired temperature. The lobe in each snowman shaped particle and the two lobes in each dumbbell shaped particle are connected to the central seed by harmonic bonds. The force constants were stiff enough to maintain the stability of lobes during all simulations. The non-bonded interactions between every pair of particles were modeled by a surface-shifted Lennard-Jones (SSLJ) potential. The functional form of the SSLJ potential between particle $i$ and particle $j$ at a distance $r$ is given by

$$
V_{\text {SSLJ }}(r)=4 \epsilon_{i j}\left[\left(\frac{\sigma}{r-\Delta}\right)^{12}-\left(\frac{\sigma}{r-\Delta}\right)^{6}\right],
$$

where $\epsilon_{i j}$ is the depth of the potential well and $\Delta=\left(\sigma_{i}+\sigma_{j}\right) / 2-1$, where $\sigma_{i}$ and $\sigma_{j}$ are the diameters of the particle $i$ and particle $j$, respectively. Here, $\sigma$ measures how close two non-bonded particles can come in an equilibrated self-assembled structure. In all of our simulations, $\epsilon_{i j}$ was set to 1.0 for every type (seedseed, lobe-lobe, seed-seed) of pair in particles. The interactions in a lobe-lobe pair are attractive, and between a seed-seed or seedlobe pair are repulsive. The cut-off distance for the pairs of lobes was set to 3.0 and the cut-off distances for the seed-seed and seedlobe pairs were set to $2^{1 / 6} \sigma$. For each set of seed diameter (except $\sigma_{S}=1.0$ ) and temperature, we carried out two simulations; in one $\sigma$ was set to 1.0 and in another one $\sigma$ was set to equal to the seed diameter, $\sigma_{S}$. In Supplementary Figure S2, we show a trace of $V_{\text {SSLJ }} v s$. $r$ at two different $\sigma$ for $\sigma_{S}=1.4$. At a higher $\sigma$, the location of the potential well shifts to the right side.

We performed a total of 165 simulations (55 simulations for each of the three $r_{\mathrm{N}}$ values; 30 simulations at $\sigma=1.0$ and 25 simulations at higher $\sigma$ values). Each simulation was carried out for $10^{8}$ steps using a time step of 0.005 . We present the potential energy per particle $v$ s. time for all systems in Supplementary Figure S3 which show a convergence of the potential energy with time. To examine the robustness of the self-assembled morphologies, we performed many additional simulations starting from different initial configurations. We observed that the self-assembled structures generated at a particular set of parameters is invariable among different starting configurations.

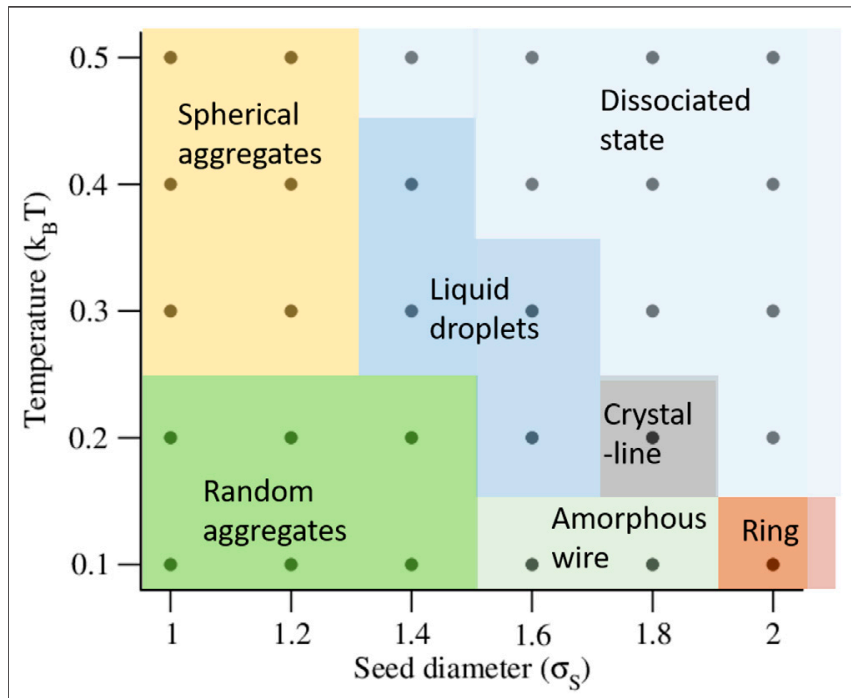

FIGURE 2 | A phase diagram of all self-assembled morphologies observed at different seed diameters and temperatures for $\sigma=1.0$ (the SSLJ parameter) and $r_{\mathrm{N}}=1.0$.

We estimated the radial distribution (RDF) functions for the seed pairs to understand the morphologies of the self-assembled structures (see details in the supporting information).

To examine the homogeneity of the self-assembled structures, we defined a parameter $\Phi$ which is given by [42].

$$
\Phi=\frac{1}{N}\left\langle\sum_{i=1}^{N} \Phi_{i}\right\rangle_{t}
$$

where $N$ is the total number of particles present in the system. $\langle\ldots\rangle$ denotes the time average, and $\Phi_{i}$ is defined as $\Phi_{i}=\left(n_{a}-n_{b}\right)^{2} /\left(n_{a}+n_{b}\right)^{2}$ where $n_{a}$ and $n_{b}$ are the number of particles of type $a$ and type $b$, respectively, that are present in the first coordination shell of particle $i$. The $\Phi_{i}$ is equal to one if all the particles present in the first coordination shell of particle $i$ are of the same type (i.e., $n_{a}$ or $n_{b}=0$ ) and the $\Phi_{i}$ is 0 if $n_{a}=n_{b}$.

\section{RESULTS AND DISCUSSION}

In this section, we describe the self-assembled morphologies and porosities obtained from the mixtures of snowman and dumbbell shaped particles at different seed diameters $\left(\sigma_{\mathrm{S}}\right)$ and temperatures $\left(k_{B} T\right)$. At first, we discuss the morphologies obtained at $\sigma=1.0$ (the SSLJ parameter) and $r_{\mathrm{N}}=1.0$. We show a state diagram in Figure 2. Then we describe the results obtained at higher $\sigma$ values and at the other number ratios of the lobed particles.

\section{Random Aggregates of Different Shapes at Lower $\sigma_{\mathbf{S}}$}

At $\sigma_{\mathrm{S}}=1.0\left(q_{\mathrm{L}}=1.0\right)$, the self-assembly occurs at all five temperatures $\left(k_{B} T=0.1,0.2,0.3,0.4,0.5\right)$ studied in this work. The RDFs shown in Figure $\mathbf{3 A}$ reveal that the self-assembled 

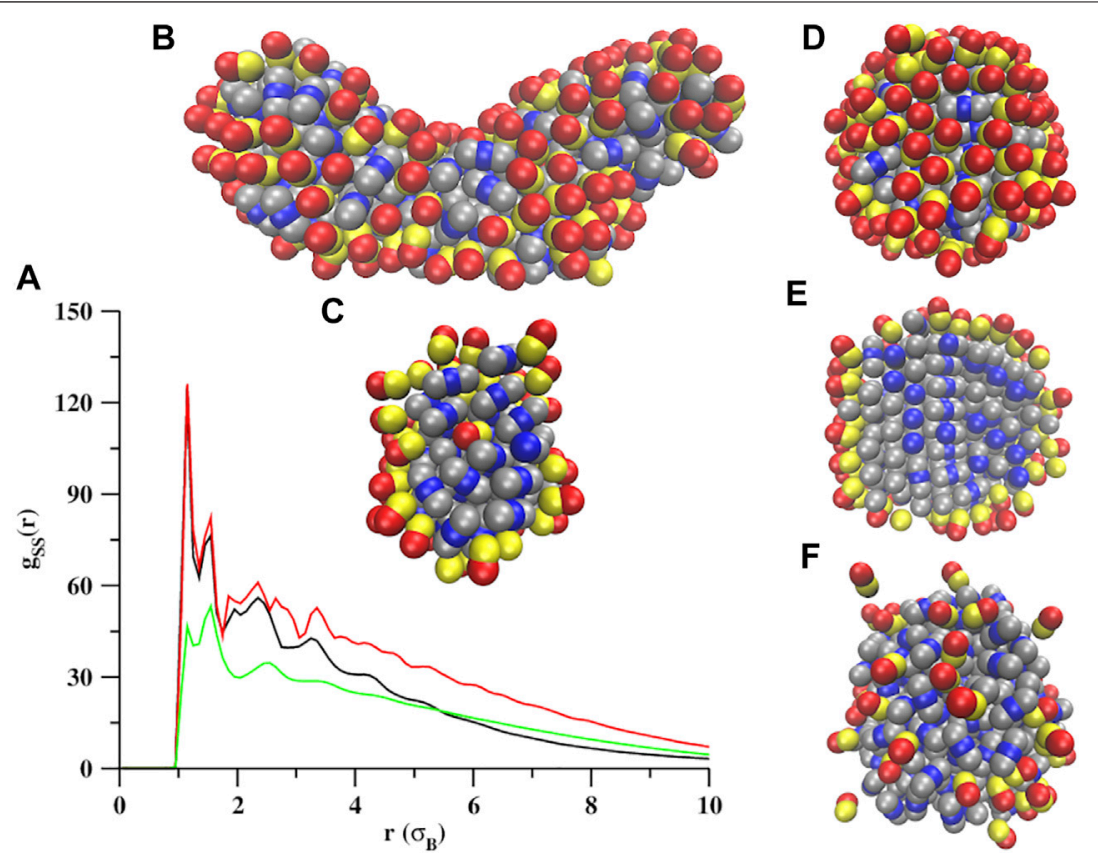

FIGURE 3 | Self-assembly of a mixture of snowman and dumbbell shaped particles at $\sigma_{S}=1.0$. The RDFs computed for the seed pairs at $k_{B} T=0.1$ (black trace), $k_{B} T=0.3$ (red trace), and $k_{B} T=0.5$ (green trace). Shown are the zoomed views of the random aggregates formed at (B) $k_{B} T=0.1$, (D) 0.3 , and (F) 0.5 . The cross-sectional views (panels $\mathbf{C}$ and $\mathbf{E}$ ) of aggregates given in $\mathbf{B}$ and $\mathbf{D}$ are also shown.

structures formed at $\sigma_{\mathrm{S}}=1.0$ are mainly random aggregates. At lower temperatures, the lobes are sticky due to stronger interactions between them. As a result, when the smaller aggregates generated at the initial stages in simulations merge to form larger aggregates, the lobed particles cannot rearrange their positions inside these aggregates, thereby leading to different shapes. The aggregates formed at lower temperatures are composed of both snowman and dumbbell shaped particles (Figure 3B). However, at higher temperatures, the lobes are not so sticky as the interactions between them are moderate, and the lobed particles can rearrange their positions to reduce the interfacial tension and finally appear in a spherical shape (Figure 2 and Figures 3D,F). The spherical aggregates formed at higher temperatures are homogeneous, as revealed by the parameter $\Phi$ at different temperatures (Figure $\mathbf{4 A}$; black curve). The cross sectional views of the random aggregates shown in Figures 3C,E reveal that the cores of the random aggregates are formed mainly by the dumbbell shaped particles and the outer most shell is covered by a single layer of snowman shaped particles. This is partly driven by the excluded volume effect and partly driven by the energy. As the volume of the dumbbell shaped particles are higher than the snowman shaped particles, the formation of the central cores of the self-assembled structure only by the dumbbell shaped particles reduces more excluded volume, which makes it favorable to form these morphologies. In addition, the dumbbell shaped particles, when they are at the cores of the self-assembled structures, utilize their two lobes to interact with the other particles, and lower the energy of the system. And the snowman shaped particles due to having one lobe interact with the other particles from one direction only, and form the outer surfaces of the self-assembled structures, making the process partly energy driven. We suggest that the self-assembled monolayers formed at lower temperatures by the snowman shaped particles can stabilize, and control several properties like wetting, adhesion, and chemical resistance of the inner self-assembled structures formed by the dumbbell shaped particles [43, 44].

We also estimated the average number of particles present in the first coordination shells of dumbbell shaped $\left(\bar{N}_{\mathrm{D}}\right)$ and snowman shaped $\left(\bar{N}_{\mathrm{S}}\right)$ particles at different seed diameters and temperatures. The changes in $\bar{N}_{\mathrm{D}}$ and $\bar{N}_{\mathrm{S}}$ with temperature for all seed diameters are shown in Figures 4B,C, respectively. At $\sigma_{\mathrm{S}}=1.0$, the values of $\bar{N}_{\mathrm{D}}$ (Figure 4B, black curve) remain nearly constant with an increase in temperature, revealing that the dumbbell shaped particles participate in the self-assembly for the entire range of temperatures. But, the values of $\bar{N}_{\mathrm{S}}$, at $\sigma_{\mathrm{S}}=1.0$ (Figure 4C, black curve) reveal that the snowman shaped particles participate in the self-assembly until $k_{B} T=0.3$, and then they begin to disassemble and at $k_{B} T=0.5$, most of the snowman shaped particles remain in a dissociated state. At $k_{B} T=0.5$, the aggregates are homogeneous, formed predominantly by the dumbbell shaped particles.

At $\sigma_{\mathrm{S}}=1.2\left(q_{\mathrm{L}}=0.83\right)$, we observed a very similar kind of selfassembly phenomenon that we observed at $\sigma_{\mathrm{S}}=1.0$ (Supplementary Figure S4). At lower temperatures $\left(k_{B} T=\right.$ $0.1,0.2)$, random aggregates of different shapes are formed (Supplementary Figures S4A,D), and at higher temperatures $\left(k_{B} T=0.3,0.4,0.5\right)$, these aggregates become spherical (Supplementary Figures. S4B,C, E-G). With an increase in temperature, the aggregates become homogeneous (Figure 4A; red curve), formed mainly by the dumbbell shaped particles. The variations in $\bar{N}_{\mathrm{D}}$ and $\bar{N}_{\mathrm{S}}$ with temperature reveal that the dumbbell shaped particles self-assemble at a wider range of 


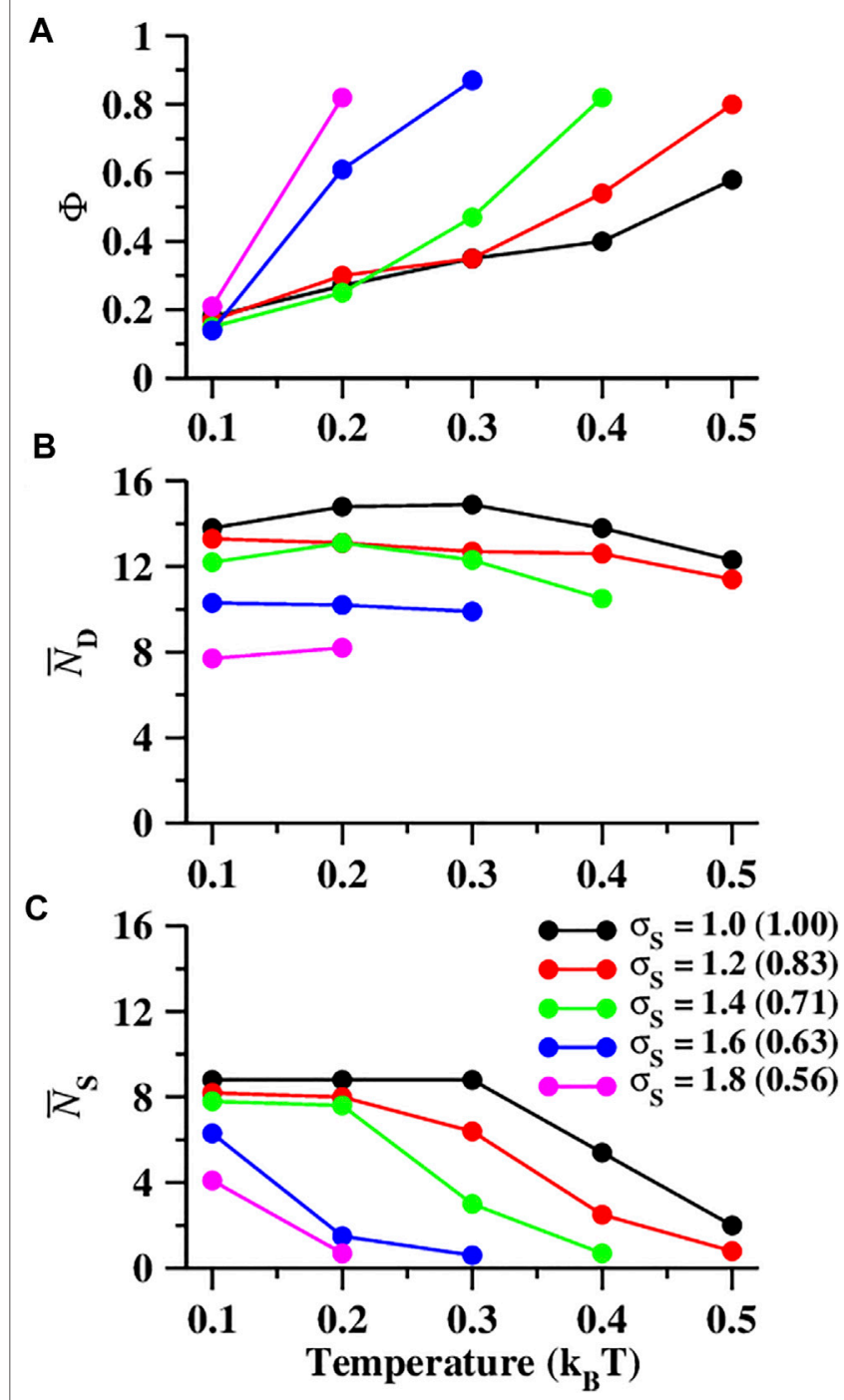

FIGURE 4 | (A) The change in the parameter $\Phi$ (defined by Eq. 2) with an increase in temperature $\left(k_{B} T\right)$ at different seed diameters $\left(\sigma_{S}\right)$ for the SSLJ parameter $\sigma=1$. At each seed diameter, the values of $\Phi$ increases with an increase in temperature, revealing the formation of homogeneous selfassembled structures at higher temperatures. (B and C) The average number of particles in the first coordination shell of (B) dumbbell shaped particles $\left(\bar{N}_{D}\right)$ and (C) snowman shaped $\left(\bar{N}_{S}\right)$ particles. The legend in the inset of panel $\mathrm{C}$ applies to all panels. The values of $q_{\mathrm{L}}$, the parameter we defined to quantify the lobe size (see Methods), are given in the parentheses.

temperatures (Figure 4B; red curve), but the snowman shaped particles start to disassemble from $k_{B} T=0.3$ and remain in a dissociated state (Figure 4C; red curve).

\section{A Transition from Random Aggregates to Liquid Droplets at Moderate $\sigma_{\mathbf{S}}$}

At $\sigma_{\mathrm{S}}=1.4\left(q_{\mathrm{L}}=0.71\right)$ and $\sigma_{\mathrm{S}}=1.6\left(q_{\mathrm{L}}=0.63\right)$, we observed a transition from random aggregates to a liquid droplet state with an increase in temperature. At $\sigma_{\mathrm{S}}=1.4$, the self-assembly occurs at $k_{B} T=0.1$ to 0.4 . At $k_{B} T=0.1$ and 0.2 , heterogeneous random aggregates form (Supplementary Figures S5A,C,E, and F). At higher temperatures $\left(k_{B} T>0.2\right)$, the self-assembled structures become homogeneous, formed predominantly by the dumbbell shaped particles (Figure 4A; green curve). At higher temperatures, the self-assembled structures formed by the dumbbell shaped particles attain a liquid droplet state (Supplementary Figures S5B,D,G), and the snowman shaped particles remain in a dissociated state.

At $\sigma_{\mathrm{S}}=1.6$, the self-assembly occurs from $k_{B} T=0.1$ to 0.3 (Figure 5 and Supplementary Figure S6). At a lower temperature, $k_{B} T=0.1$, we observed wire-like structures, that are amorphous and heterogeneous in nature, formed by both types (dumbbell and snowman shaped) of particles (Figure 5B and Supplementary Figure S6B). At $k_{B} T=0.2$, most of the snowman shaped particles disassemble (Figure 4C, blue curve) and the dumbbell shaped particles self-assemble into amorphous three-dimensional aggregates (Figure 5C and Supplementary Figure S6C). At both $k_{B} T=0.1$ and 0.2 , the dumbbell shaped particles form the cores of the self-assembled structures and the snowman shaped particles are found in the outer shell (Figures 5B,C). At both temperatures, the average number of particles present in the first coordination shells of the dumbbell shaped particles $\left(\bar{N}_{\mathrm{D}}\right)$ is 10 (Figure $\mathbf{4 B}$; blue curve), where each of the two lobes are attached with five other particles (Figures 5E,F). From the top-view, the first coordination shell resembles a starfish (Figure 5E). At a higher temperature $\left(k_{B} T=0.3\right)$, mainly the dumbbell shaped particles participate in the selfassembly, and form a liquid droplet state (Figure 5D and Supplementary Figure S6D).

For $\sigma_{\mathrm{S}} \geq 1.6$, we observed a rapid phase separation with an increase in temperature. For an increase in temperature $\left(k_{B} T\right)$ from 0.1 to 0.2 at $\sigma_{\mathrm{S}}=1.6$, the values of $\Phi$ were observed to significantly increase from 0.14 to 0.61 (Figure 4A; blue curve). The values of $\bar{N}_{\mathrm{S}}$ (i.e., the average number of particles in the first coordination shell of the snowman shaped particles) were also observed to undergo a significant decrease from 6.3 to 1.5 with an increase in temperature from 0.1 to 0.2 (Figure 4C; blue curve), while the values of $\bar{N}_{\mathrm{D}}$ remain constant at around 8.5 for the entire range of temperature (Figure 4B; blue curve). These data establish the fact that the snowman shaped particles get separated from the aggregates rapidly at higher seed diameters than they do at lower seed diameters. A similar phase separation phenomenon was observed at $\sigma_{\mathrm{S}}=1.8$.

\section{Amorphous Wire and Ring like Structures and Crystalline Structures at higher $\sigma_{\mathrm{S}}$}

At $\sigma_{\mathrm{S}}=1.8$, the self-assembly occurs only at lower temperatures $\left(k_{B} T=0.1\right.$ and 0.2$)$, and a transition from a wire-like structure (Figure 6B and Supplementary Figure S7B) to a crystalline structure (Figure 6D and Supplementary Figure S7C) is observed with an increase in temperature. At $k_{B} T=0.1$, the heterogeneous wire-like structures (Figure $\mathbf{6 B}$ and Supplementary Figure S7B) are formed by both types (snowman and dumbbell shaped) of particles. The lobed particles were observed to self-assemble into tetrahedral or octahedral clusters (Figure 6C) before forming an extended 


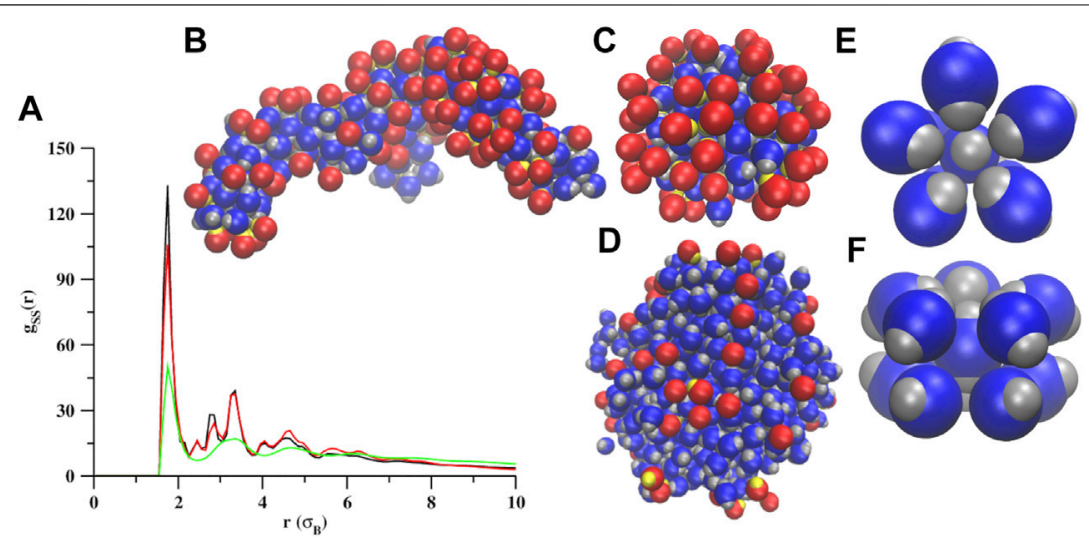

FIGURE 5 | Self-assembly of a mixture of snowman and dumbbell shaped particles at $\sigma_{\mathrm{S}}=1.6$. (A) The RDFs computed for the seed pairs at $k_{B} T=0.1$ (black trace), $k_{B} T=0.2$ (red trace), and $k_{B} T=0.3$ (green trace). Shown also are the enlarged views of a wire-like cluster formed at $k_{B} T=0.1$ (B), a three-dimensional amorphous cluster (C), and a liquid droplet (D). In all of these structures, the snowman shaped particles are found at the surfaces only. The first coordination shells of the dumbbell shaped particles look similar at $k_{B} T=0.1$ and $k_{B} T=0.2$. The top and side views of the first coordination shell are shown in panels (E,F).

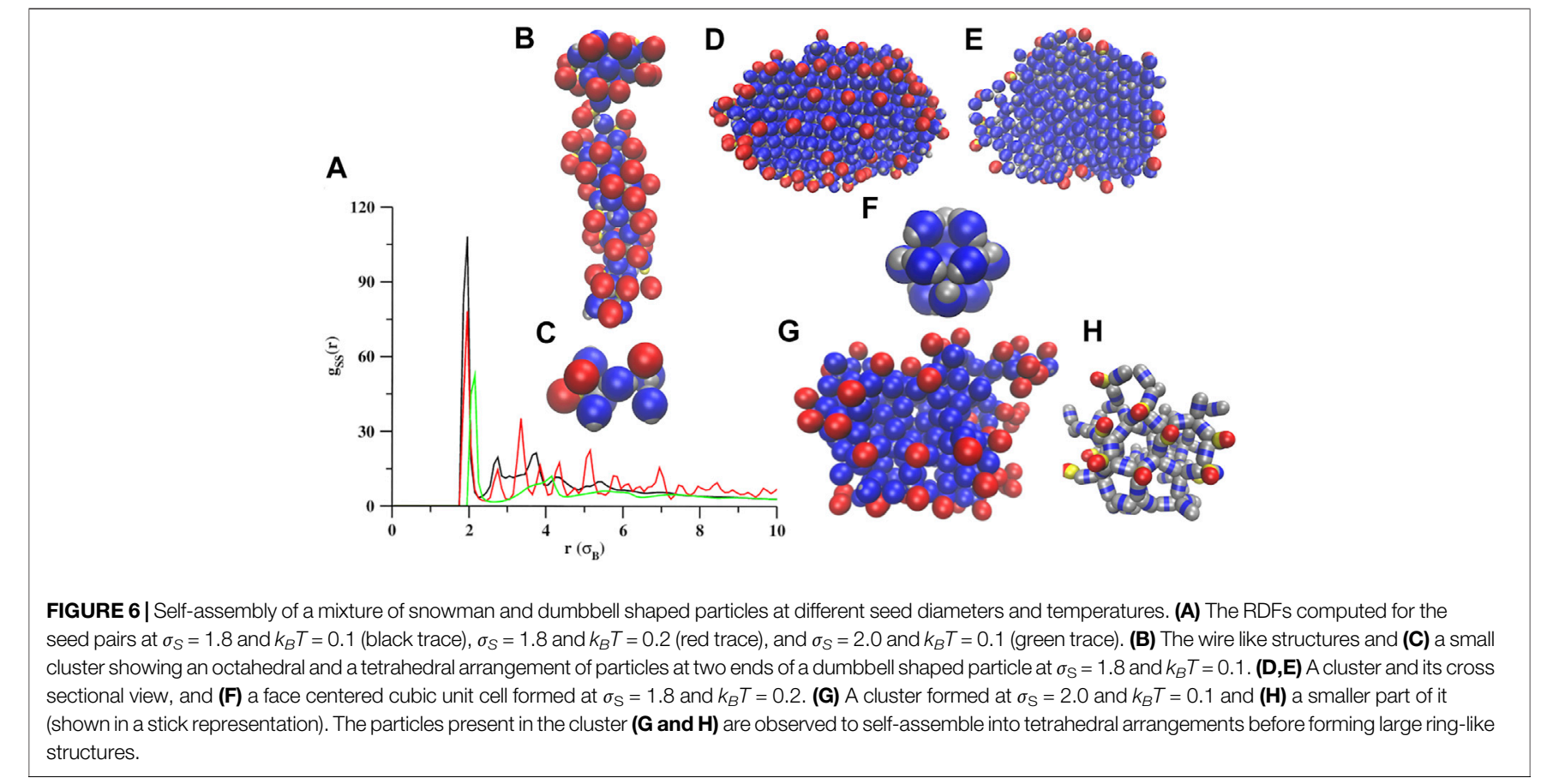

wire-like structure. The dumbbell shaped particles contribute to form the central cores of the wires, thereby helping in lengthening the wires via two lobes at the opposite poles. The snowman shaped particles are mainly found at the surfaces of wires, which prevents the growth of wires orthogonal to the axes of the wires. At higher seed diameters, the volumes of the snowman and the dumbbell shaped particles are almost equal. Therefore, the excluded volume effect almost diminishes at this condition. So, the formation of the central cores of the self-assembled structures by the dumbbell shaped particles and the outer surface by the snowman shaped particles is mainly energy driven.
At higher temperatures, the snowman shaped particles separate from the assembly leaving only homogeneous selfassembled structures formed by the dumbbell shaped particles. We observed a large increase in the $\Phi$ value from 0.21 to 0.82 (Figure 4A; the magenta curve) with a small increment in the temperature from 0.1 to 0.2 , revealing a rapid transition from the heterogeneous to homogeneous structures. The variations in $\bar{N}_{\mathrm{D}}$ (Figure 4B; the magenta curve) and $\bar{N}_{\mathrm{S}}$ (Figure 4C; the magenta curve) values with temperature also support the observation of a rapid phase separation. The self-assembled structures formed at $k_{B} T=0.2$ are crystalline where the unit cell is a face-centered cubic lattice (Figure $6 \mathbf{F}$ and 


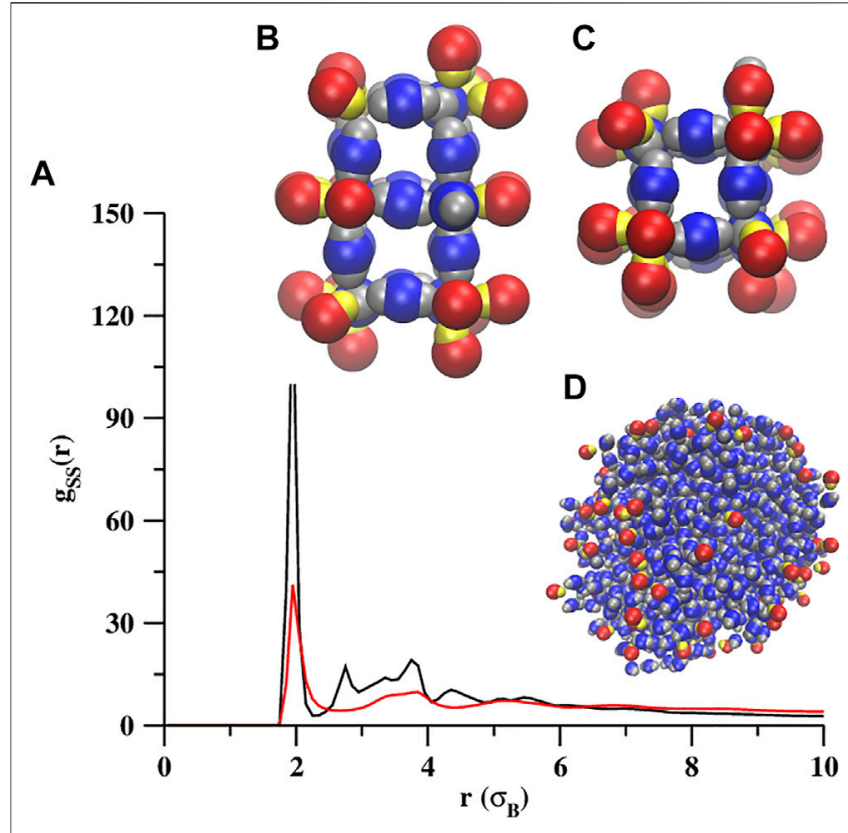

FIGURE 7 | Self-assembly of a binary mixture of snowman and dumbbell shaped particles at $\sigma_{\mathrm{S}}=1.4$ and $\sigma=1.4$. Shown are the RDFs for the wire like structures (black trace) formed at $k_{B} T=0.1$ and liquid droplets (red trace) formed at $k_{B} T=0.2$. The top and side views of a part of wirelike structures $(\mathbf{B}, \mathbf{C})$ and a liquid droplet (D) are also shown.

Supplementary Figure S7D). In Figures 6D,E, we show a selfassembled cluster and its cross sectional view formed at $k_{B} T=$ 0.2 . It reveals that the bulk of the crystalline structures is constituted by the dumbbell shaped particles only, and few lattice points at the surfaces of the crystalline structures are occupied by the snowman shaped particles (the red spheres).

At $\sigma_{\mathrm{S}}=2.0$, the self-assembly occurs only at $k_{B} T=0.1$, predominantly by the dumbbell shaped particles (Figure 6G). The self-assembled structures are amorphous and made up of ring like structures (Figure $\mathbf{6 H}$ ). We did not observe any longrange order between the particles (Figure 6A; the green curve) but a short-range order is observed where the first coordination shell possesses a trigonal prismatic geometry. The lobed particles were observed to self-assemble into a tetrahedral geometry before forming a ring like structure (Figure 6G). The dumbbell shaped particles form the bulk of the clusters, and the snowman shaped particles are observed only at the surfaces of the clusters (Figure 6G).

\section{Heterogeneous Wire-like Structures to Homogeneous Liquid Droplets at $\sigma_{\mathrm{S}}=1.4$ and $\sigma=1.4$}

The self-assembled morphologies, that we have described so far, were obtained at the SSLJ parameter $\sigma=1.0$. Now we will describe the self-assembled morphologies obtained at higher $\sigma$. For the seed diameters $\left(\sigma_{\mathrm{S}}\right)$ equal to $1.2,1.4,1.6,1.8$, and 2.0 , we carried out a second set of simulations where the values of $\sigma$ were set equal to their respective $\sigma_{\mathrm{S}}$ values. At these higher $\sigma$ values, we observed the self-assembly only at $\sigma_{S}=1.2$ and 1.4. At $\sigma_{\mathrm{S}}=1.2$, we observed self-assembly at four different temperatures $\left(k_{B} T=0.1\right.$, $0.2,0.3$, and 0.4 ). At lower temperatures, heterogeneous random aggregates are formed, and at higher temperatures, homogeneous liquid droplets primarily formed by the dumbbell shaped particles are observed (Supplementary Figure S8). At $\sigma_{\mathrm{S}}=1.4$, we observed the self-assembly at two different temperatures $\left(k_{B} T=0.1\right.$ and 0.2$)$. At $k_{B} T=0.1$, the heterogeneous wire-like structures (Figure 7A), that are amorphous in nature, are formed. The lobed particles self-assemble into octahedral clusters (Figures 7B,C) before forming the extended wire-like structures. At $k_{B} T=0.2$, homogeneous liquid droplets are formed mainly by the dumbbell shaped particles, and the

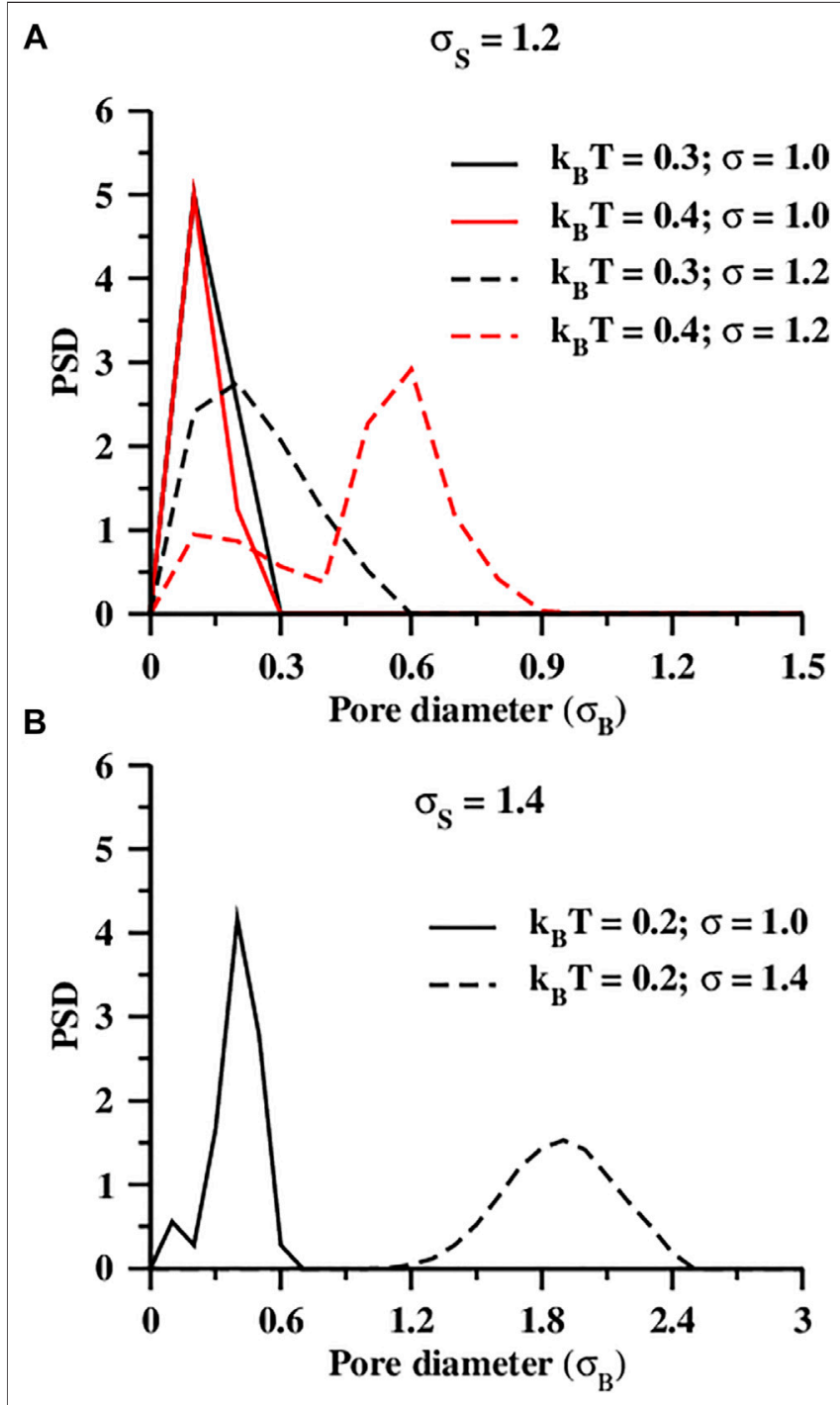

FIGURE 8|Pore size distributions (PSDs) at different temperatures and at different values of the SSLJ parameter, $\sigma$ for the seed diameter, $\sigma_{\mathrm{S}}=1.2$ (A) and 1.4 (B). For comparison, we present the PSDs only at those temperatures, where three-dimensional self-assembled structures are formed for both the $\sigma$ values. 
snowman shaped particles are found in a dissociated state (Figure 7D).

At any seed diameter and temperature, the self-assembled morphologies remain same at different number ratios $\left(r_{N}\right)$ of the lobed particles. At any combination of the seed diameter $\left(\sigma_{S}\right)$, temperature $\left(k_{B} T\right)$, and the SSLJ parameter, $\sigma$, the morphologies of the self-assembled structures formed at two other number ratios $\left(r_{N}=0.5\right.$ and 2.0) of the lobed particles are similar to the structures formed at $r_{N}=1.0$ (Supplementary Figures S9, S10). At any seed diameters, homogeneous structures are formed at lower temperatures, and heterogeneous structures are formed at higher temperatures (Supplementary Figure S11). The variations in $\bar{N}_{\mathrm{D}}$ and $\bar{N}_{\mathrm{S}}$ (Supplementary Figure S12) with an increase in temperature are also similar to the variations that we observed in the case of $r_{N}=1.0$ (Figure 4). The values of $\bar{N}_{\mathrm{D}}$ (Supplementary Figures S12A,B) remain almost same at the entire range of temperature. But, the values of $\bar{N}_{S}$ (Supplementary Figures S12C,D) start to decrease after a certain temperature at any seed diameter as the snowman shaped particles remain in a dissociated state at higher temperatures. At $r_{N}=2.0$, as the number of the snowman shaped particles is twice the number of the dumbbell shaped particles, the selfassembled clusters formed at $r_{N}=2.0$ are fully wrapped by the snowman shaped particles. In contrast, at $r_{N}=0.5$, the clusters are partially wrapped due to the less number of snowman shaped particles in the mixture.

\section{Porosities of Self-Assembled Structures}

At the SSLJ patameter $\sigma=1.0$, the largest pores are found at $\sigma_{\mathrm{S}}=$ 2.0 and $k_{B} T=0.1$. We estimated pore diameters for those structures that are three dimensional, and not extended wirelike. At any seed diameter, the three-dimensional structures usually form at higher temperatures, and the extended wire-like structures or narrow random aggregates form at lower temperatures. Therefore we estimated the pore size distributions, which are shown in Figure 8, Supplementary Figures S13, S14, for the higher temperature structures only. For three-dimensional self-assembled structures, we extracted large cuboids, and then used the Zeo++ software $[45,46]$ to estimate the pore size distributions, as carried out in our previous work [30,31]. For each seed diameter, the pore diameters were observed to increase with an increase in the temperature. The lobed particles having larger seeds or smaller lobes were observed to produce bigger pores than the particles having smaller seeds. At $\sigma=1.0$, the largest pores were found for $\sigma_{\mathrm{S}}=2.0$ at $k_{B} T=0.1$ (Supplementary Figure S13F). We obtained a broader pore size distribution because of the amorphous ring structures formed at this condition. The pore diameters were found to vary between $\sim 1.5 \sigma_{\mathrm{B}}$ and $\sim 3.5 \sigma_{B}$.

\section{Self-Assembled Structure Formed at a Higher $\sigma$ Value Produce Larger Pores than the Corresponding Structure Formed at a Lower Sigma Value}

The self-assembled structures formed at higher $\sigma$ values are observed to form larger pores than the corresponding structures formed at lower $\sigma$ values (Figure 8). Among the self-assembled structures formed at higher $\sigma$ values, the structures self-assembled at $\sigma_{\mathrm{S}}=1.4$ and $\sigma=1.4$ were found to possess the largest pores at $k_{B} T=0.2$ (Figure 8B, Supplementary Figure S14B). At this condition, the pore diameter varies between $\sim 1.0 \sigma_{\mathrm{B}}$ and $\sim$ $2.5 \sigma_{\mathrm{B}}$. The structures self-assembled at the same seed diameter and temperature $\left(\sigma_{\mathrm{S}}=1.4\right.$ and $\left.k_{B} T=0.2\right)$ but at a lower $\sigma$ value $(\sigma=$ $1.0)$ produce very small pores $\left(\sim 0.2 \sigma_{\mathrm{B}}\right.$ to $\left.\sim 0.7 \sigma_{\mathrm{B}}\right)$, revealing the effect of the Lennard-Jones parameters $\sigma$ on the porosities of the self-assembled structures. The central seeds, at a higher $\sigma$ value, become more repulsive and therefore remain further from each other compared to a lower $\sigma$, which is why the pore diameters at a higher $\sigma$ are much larger than at a lower $\sigma$.

As all the simulation domains in our work contain a mixture of snowman and dumbbell shaped particles, the self-assembly phenomenon is observed mainly at lower temperatures. For example, in our earlier study [31], we observed that the highest temperature at which the dumbbell shaped particles of $\sigma_{\mathrm{S}}=1.6$ selfassemble is 0.4 , and at that condition the pore diameters vary between $0.1 \sigma_{\mathrm{B}}$ and $2.3 \sigma_{\mathrm{B}}$ [31]. But in the presence of snowman shaped particles, the highest temperature at which the self-assembly occurs at $\sigma_{S}=1.6$ is 0.3 . Although the self-assembled structures formed at $k_{B} T=0.3$ in the present work are almost similar to the structures formed at $k_{B} T=0.4$ in our previous study (in both cases liquid droplets are formed by the dumbbell shaped particles), the pore diameters found in the present work (Supplementary Figure S13D, red curve) are relatively smaller $\left(0.1 \sigma_{B}\right.$ to $\left.1.2 \sigma_{B}\right)$ than the previous work due to the difference in the temperature. As pore diameters increase with the temperature, and the presence of the snowman shaped particles inhibits the self-assembly at higher temperatures, we recommend the usage of only dumbbell shaped particles for better porous self-assembled structures. Moreover, as the snowman shaped particles remain only at surfaces of the selfassembled structures and the bulk of the self-assembled structures are formed mainly by the dumbbell shaped particles at any seed diameter and temperature, the snowman shaped particles do not have a significant impact on the porosities of the self-assembled structures.

\section{CONCLUSION}

We have reported studies on self-assembled structures obtained from a mixture of the snowman and dumbbell shaped particles at different lobe sizes and temperatures. We also investigated the effect of the SSLJ parameter $\sigma$ on the self-assembled morphologies and porosites. At lower temperatures, heterogeneous self-assembled structures are formed by both the dumbbell and snowman shaped particles. The dumbbell shaped particles form the cores of self-assembled structures, and the snowman shaped particles are self-assembled in a single layer at the surfaces of the self-assembled structures. With an increase in temperature, homogeneous selfassembled structures formed predominantly by the dumbbell shaped particles are observed, and the snowman shaped particles are found in a dissociated state. The difference in the number of lobes between these two types of particles is the reason for the selfassembly of snowman particles only at lower temperatures, while 
those of the dumbbell shaped particles at higher temperatures too. At larger seed diameters, the transition from the heterogeneous to homogeneous structures occurs faster than at smaller seed diameters. Depending on the seed diameter and temperature, we found different types of self-assembled structures (random aggregates, wire-like structures, liquid droplets, crystalline, and ring structures). The self-assembled morphologies are invariant with respect to the number ratios of the constituent lobed particles in the mixture. At higher $\sigma$ values, the self-assembly occurs only at lower seed diameters $\left(\sigma_{S}=1.2\right.$ and 1.4). Our work suggests that the repulsive interactions between the seed particles play a major role in producing porous self-assembled structures, as suggested by the results at various $\sigma$ values. Our work also shows that the simpler particle shapes like snowman and dumbbell shaped lobed particles, having very small number of lobes that are easier to synthesize, can produce a variety of structures when they are mixed and when the interactions between them are tuned.

\section{DATA AVAILABILITY STATEMENT}

The original contributions presented in the study are included in the article/Supplementary Material, further inquiries can be directed to the corresponding author.

\section{REFERENCES}

1. Manoharan VN. Colloidal matter: Packing, geometry, and entropy. Science (2015) 349:1253751. doi:10.1126/science.1253751

2. Zhang Z, and Glotzer SC. Self-assembly of patchy particles. Nano Lett (2004) 4 : 1407-13. doi:10.1021/nl0493500

3. Chen Q, Bae SC, and Granick S. Directed self-assembly of a colloidal kagome lattice. Nature (2011) 469:381-4. doi:10.1038/nature09713

4. Kraft DJ, Ni R, Smallenburg F, Hermes M, Yoon K, Weitz DA, et al. Surface roughness directed self-assembly of patchy particles into colloidal micelles. Proc Natl Acad Sci (2012) 109:10787-92. doi:10.1073/pnas.1116820109

5. Wang Y, Wang Y, Breed DR, Manoharan VN, Feng L, Hollingsworth AD, et al. Colloids with valence and specific directional bonding. Nature (2012) 491: 51-5. doi:10.1038/nature11564

6. Ku KH, Kim Y, Yi G-R, Jung YS, and Kim BJ. Soft patchy particles of block copolymers from interface-engineered emulsions. ACS Nano (2015) 9: 11333-41. doi:10.1021/acsnano.5b05058

7. Diaz A. JA, Oh JS, Yi G-R, and Pine DJ. Photo-printing of faceted dna patchy particles. Proc Natl Acad Sci U.S.A (2020) 117:10645-53. doi:10.1073/pnas.1918504117

8. Pons-Siepermann IC, and Glotzer SC. Design of patchy particles using quaternary self-assembled monolayers. ACS Nano (2012) 6:3919-24. doi: $10.1021 / \mathrm{nn} 300059 \mathrm{x}$

9. Long AW, and Ferguson AL. Rational design of patchy colloidsvialandscape engineering. Mol Syst Des Eng (2018) 3:49-65. doi:10.1039/c7me00077d

10. Long AW, and Ferguson AL. Nonlinear machine learning of patchy colloid self-assembly pathways and mechanisms. J Phys Chem B (2014) 118:4228-44. doi:10.1021/jp500350b

11. Morphew D, Shaw J, Avins C, and Chakrabarti D. Programming hierarchical self-assembly of patchy particles into colloidal crystals via colloidal molecules. ACS Nano (2018) 12:2355-64. doi:10.1021/acsnano.7b07633

12. Tsyrenova A, Miller K, Yan J, Olson E, Anthony SM, and Jiang S. Surfactantmediated assembly of amphiphilic janus spheres. Langmuir (2019) 35: 6106-11. doi:10.1021/acs.langmuir.9b00500

13. Kraft DJ, Vlug WS, van Kats CM, van Blaaderen A, Imhof A, and Kegel WK. Self-assembly of colloids with liquid protrusions. J Am Chem Soc (2009) 131: 1182-6. doi:10.1021/ja8079803

\section{AUTHOR CONTRIBUTIONS}

HV: conceptualization, supervision, and funding acquisition. SP: modeling, simulation, analysis. SP and HV: writing, draft preparation. All authors contributed to the article and approved the submitted version.

\section{FUNDING}

We are grateful for financial support provided by the National Science Foundation (award No. OIA-1757371; HV). We acknowledge computational support through the following resources: Premise, a central shared HPC cluster at UNH supported by the Research Computing Center; and BioMade, a heterogeneous CPU/GPU cluster supported by the NSF EPSCoR award (OIA-1757371; HV).

\section{SUPPLEMENTARY MATERIAL}

The Supplementary Material for this article can be found online at: https://www.frontiersin.org/articles/10.3389/fphy.2021.767623/ full\#supplementary-material

14. Hong L, Cacciuto A, Luijten E, and Granick S. Clusters of amphiphilic colloidal spheres. Langmuir (2008) 24:621-5. doi:10.1021/la7030818

15. Bharti B, Rutkowski D, Han K, Kumar AU, Hall CK, and Velev OD. Capillary bridging as a tool for assembling discrete clusters of patchy particles. J Am Chem Soc (2016) 138:14948-53. doi:10.1021/jacs.6b08017

16. Sato M. Effect of patch area and interaction length on clusters and structures formed by one-patch particles in thin systems. ACS Omega (2020) 5:28812-22. doi:10.1021/acsomega.0c04159

17. Sato M. Effect of the interaction length on clusters formed by spherical onepatch particles on flat planes. Langmuir (2021) 37:4213-21. doi:10.1021/ acs.langmuir.1c00102

18. Harper ES, van Anders G, and Glotzer SC. The entropic bond in colloidal crystals. Proc Natl Acad Sci USA (2019) 116:16703-10. doi:10.1073/ pnas. 1822092116

19. van Anders G, Klotsa D, Ahmed NK, Engel M, and Glotzer SC. Understanding shape entropy through local dense packing. Proc Natl Acad Sci USA (2014) 111:E4812-E4821. -E4821 doi:10.1073/pnas.1418159111

20. Ravaine S, and Duguet E. Synthesis and assembly of patchy particles: Recent progress and future prospects. Curr Opin Colloid Interf Sci (2017) 30:45-53. doi:10.1016/j.cocis.2017.05.002

21. Li F, Josephson DP, and Stein A. Colloidal assembly: The road from particles to colloidal molecules and crystals. Angew Chem Int Ed (2011) 50:360-88. doi:10.1002/anie.201001451

22. Glotzer SC, Solomon MJ, and Kotov NA. Self-assembly: From nanoscale to microscale colloids. Aiche J (2004) 50:2978-85. doi:10.1002/aic.10413

23. Xia Y, Gates B, and Li Z-Y. Self-assembly approaches to three-dimensional photonic crystals. Adv Mater (2001) 13:409-13. doi:10.1002/15214095(200103)13:6<409:aid-adma409>3.0.co;2-c

24. Pawar AB, and Kretzschmar I. Fabrication, assembly, and application of patchy particles. Macromol Rapid Commun (2010) 31:150-68. doi:10.1002/ marc.200900614

25. Boncheva M, and Whitesides GM. Biomimetic Approaches to the Design of Functional, Self-Assembling Systems. 1st ed. edn, vol. 1. New York, NY: Marcel Dekker (2004). p. 287-94. 873.

26. Bao H, Bihr T, Smith A-S, and Klupp Taylor RN. Facile colloidal coating of polystyrene nanospheres with tunable gold dendritic patches. Nanoscale (2014) 6:3954-66. doi:10.1039/c3nr04016j 
27. Meester V, Verweij RW, van der Wel C, and Kraft DJ. Colloidal recycling: Reconfiguration of random aggregates into patchy particles. ACS Nano (2016) 10:4322-9. doi:10.1021/acsnano.5b07901

28. van Ravensteijn BGP, Kamp M, van Blaaderen A, and Kegel WK. General route toward chemically anisotropic colloids. Chem Mater (2013) 25:4348-53. doi: $10.1021 / \mathrm{cm} 4025606$

29. Gröschel AH, Walther A, Löbling TI, Schacher FH, Schmalz H, and Müller AHE. Guided hierarchical co-assembly of soft patchy nanoparticles. Nature (2013) 503:247-51. doi:10.1038/nature12610

30. Paul S, and Vashisth H. Self-assembly of lobed particles into amorphous and crystalline porous structures. Soft Matter (2020) 16:1142-7. doi:10.1039/c9sm01878f

31. Paul S, and Vashisth H. Self-assembly behavior of experimentally realizable lobed patchy particles. Soft Matter (2020) 16:8101-7. doi:10.1039/d0sm00954g

32. Rocha BC, Paul S, and Vashisth H. Enhanced porosity in self-assembled morphologies mediated by charged lobes on patchy particles. J Phys Chem B (2021) 125:3208-15. doi:10.1021/acs.jpcb.0c11096

33. Gorai B, Rocha BC, and Vashisth H. Design of functionalized lobed particles for porous self-assemblies. JOM (2021) 73:2413-22. doi:10.1007/s11837-021-04715-w

34. Rovigatti L, de las Heras D, Tavares JM, Telo da Gama MM, and Sciortino F. Computing the phase diagram of binary mixtures: A patchy particle case study. J Chem Phys (2013) 138:164904. doi:10.1063/1.4802026

35. Park J-G, Forster JD, and Dufresne ER. High-yield synthesis of monodisperse dumbbell-shaped polymer nanoparticles. J Am Chem Soc (2010) 132:5960-1. doi:10.1021/ja101760q

36. Peng B, Vutukuri HR, van Blaaderen A, and Imhof A. Synthesis of fluorescent monodisperse non-spherical dumbbell-like model colloids. J Mater Chem (2012) 22:21893-900. doi:10.1039/c2jm35229j

37. Matson JB, Zha RH, and Stupp SI. Peptide self-assembly for crafting functional biological materials. Curr Opin Solid State Mater Sci (2011) 15:225-35. doi:10.1016/j.cossms.2011.08.001

38. Loh QL, and Choong C. Three-dimensional scaffolds for tissue engineering applications: Role of porosity and pore size. Tissue Eng B: Rev (2013) 19: 485-502. doi:10.1089/ten.teb.2012.0437

39. Avvisati G, Vissers T, and Dijkstra M. Self-assembly of patchy colloidal dumbbells. J Chem Phys (2015) 142:084905. doi:10.1063/1.4913369

40. Anderson JA, Lorenz CD, and Travesset A. General purpose molecular dynamics simulations fully implemented on graphics processing units. J Comput Phys (2008) 227:5342-59. doi:10.1016/j.jcp.2008.01.047
41. Glaser J, Nguyen TD, Anderson JA, Lui P, Spiga F, Millan JA, et al. Strong scaling of general-purpose molecular dynamics simulations on gpus. Comp Phys Commun (2015) 192:97-107. doi:10.1016/j.cpc.2015.02.028

42. Goyal A, Hall CK, and Velev OD. Self-assembly in binary mixtures of dipolar colloids: Molecular dynamics simulations. J Chem Phys (2010) 133:064511. doi: $10.1063 / 1.3477985$

43. Laibinis PE, Whitesides GM, Allara DL, Tao YT, Parikh AN, and Nuzzo RG. Comparison of the structures and wetting properties of selfassembled monolayers of $\mathrm{n}$-alkanethiols on the coinage metal surfaces, copper, silver, and gold. J Am Chem Soc (1991) 113:7152-67. doi:10.1021/ ja00019a011

44. Love JC, Estroff LA, Kriebel JK, Nuzzo RG, and Whitesides GM. Selfassembled monolayers of thiolates on metals as a form of nanotechnology. Chem Rev (2005) 105:1103-70. doi:10.1021/cr0300789

45. Pinheiro M, Martin RL, Rycroft $\mathrm{CH}$, and Haranczyk M. High accuracy geometric analysis of crystalline porous materials. CrystEngComm (2013) 15:7531-8. doi:10.1039/c3ce41057a

46. Pinheiro M, Martin RL, Rycroft CH, Jones A, Iglesia E, and Haranczyk M. Characterization and comparison of pore landscapes in crystalline porous materials. J Mol Graphics Model (2013) 44:208-19. doi:10.1016/ j.jmgm.2013.05.007

Conflict of Interest: The authors declare that the research was conducted in the absence of any commercial or financial relationships that could be construed as a potential conflict of interest.

Publisher's Note: All claims expressed in this article are solely those of the authors and do not necessarily represent those of their affiliated organizations, or those of the publisher, the editors and the reviewers. Any product that may be evaluated in this article, or claim that may be made by its manufacturer, is not guaranteed or endorsed by the publisher.

Copyright $(02021$ Paul and Vashisth. This is an open-access article distributed under the terms of the Creative Commons Attribution License (CC BY). The use, distribution or reproduction in other forums is permitted, provided the original author(s) and the copyright owner(s) are credited and that the original publication in this journal is cited, in accordance with accepted academic practice. No use, distribution or reproduction is permitted which does not comply with these terms. 